Pacific

Journal of

Mathematics

\title{
COLORING MAPS OF PERIOD THREE
}

J.M. Aarts, R.J. Fokkink, AND J. Vermeer 


\title{
COLORING MAPS OF PERIOD THREE
}

\author{
J.M. Aarts, R.J. FoKkInK, And J. VermeER
}

We investigate the color number and genus for fixed-point free maps of order three. A result that has the flavor of the Ljusternik-Schnirelmann theorem for involutions is established. The $Y$-sphere, the combinatorial boundary of the product of tripods, is studied in detail. Problems of coloring non-invariant subspaces are touched upon.

\section{Introduction.}

All spaces are assumed to be separable metric and all mappings are assumed to be continuous.

Definition 1. Suppose that $f: X \rightarrow X$ is a map from $X$ to itself. An open subset $B$ of $X$ is called a color of $(X, f)$ if $f(B) \cap B=\emptyset$ or, equivalently, $f^{-1}(B) \cap B=\emptyset$. A coloring of $(X, f)$ is a finite cover $\mathcal{B}$ of $X$ consisting of colors. The minimal cardinality of a coloring $\mathcal{B}$ is called the color number $\operatorname{col}(X, f)$ of $(X, f)$.

In the definition of color we could have used closed subsets as well. By shrinking an open coloring a closed coloring may be obtained and the colors of a closed coloring can be enlarged so as to obtain an open coloring. The situation is more delicate when considering non-invariant subspaces, as was shown in [8]. For a fixed-point free homeomorphism $f$ of an $n$-dimensional space $X$ we have $\operatorname{col}(X, f) \leq n+3$; if moreover the map $f$ is an involution then $\operatorname{col}(X, f) \leq n+2,[3]$.

In this paper we study fixed-point free maps $\sigma: X \rightarrow X$ of order 3, i.e., $\sigma^{3}(x)=x$ for each $x \in X$. It is to be noted that for a color $B$ of $(X, \sigma)$, when $\sigma$ has period 3, the sets $B, \sigma(B)$ and $\sigma^{2}(B)$ are pairwise disjoint. One of the reasons for studying maps of period 3 is that they provide examples of pairs $(X, \sigma)$ with $\operatorname{dim}(X)=n$ and $\operatorname{col}(X, \sigma)=n+3$. A second reason is that there is an intimate relation between the color number and the genus, which we now define.

Definition 2. Let $X$ be a space and $\sigma: X \rightarrow X$ a map of period 3 without fixed-points.

(1) A subset $B$ of $X$ is called a set of first type if there exists a color $C$ of $(X, \sigma)$ such that $B=C \cup \sigma(C) \cup \sigma^{2}(C)$; we also say that $B$ is generated by $C$. 
(2) We say that the genus of the space $X$ is at most $k$ if $X$ can be written as a union of $k$ sets of first type. Notation: $\operatorname{gen}(X, \sigma) \leq k$.

Recall that if $f: X \rightarrow X$ and $g: Y \rightarrow Y$ are mappings then a mapping $h: X \rightarrow Y$ is said to be equivariant if $h \circ f=g \circ h$. Note that for an equivariant $h: X \rightarrow Y$ and mappings $f$ and $g$ of order 3 we have $\operatorname{col}(X, f) \leq \operatorname{col}(Y, g)$ and $\operatorname{gen}(X, f) \leq \operatorname{gen}(Y, g)$.

We now formulate the main results, which are generalizations of the Ljusternik-Schnirelmann theorem for involutions on $S^{n}$.

Theorem 1. Let $X$ be a space and $\sigma: X \rightarrow X$ a map of period 3 without fixed-points. If $\operatorname{gen}(X, \sigma)=n+1$ and if $A_{1}, \ldots, A_{n+1}$ are colors of $X$ with $X=\bigcup_{i=1}^{n+1}\left[A_{i} \cup \sigma\left(A_{i}\right) \cup \sigma^{2}\left(A_{i}\right)\right]$ then

$$
\bigcap_{i=1}^{n+1}\left[A_{i} \cup \sigma\left(A_{i}\right)\right] \neq \emptyset .
$$

Theorem 2. Let $X$ be a space and $\sigma: X \rightarrow X$ a map of period 3 without fixed-points. If $\operatorname{col}(X, \sigma)=n+3$ and $\left\{A_{1}, \ldots, A_{n+1}, A_{n+2}, A_{n+3}\right\}$ is a coloring of $X$ then

$$
\bigcap_{i=1}^{n+1}\left[A_{i} \cup \sigma\left(A_{i}\right)\right] \neq \emptyset .
$$

We shall also obtain a bound on the color number of non-invariant subspaces, using the results of $[\mathbf{3}]$. We shall use the results above to show that this bound is almost sharp. Theorem 1 can be extended to general periodic maps on paracompact spaces [1] by a different proof. The proof in the present paper concerns certain universal spaces, the $Y$-cube and the $Y$-sphere, which are interesting in their own right.

\section{The $Y$-cube $Y^{n}$ and the $Y$-sphere $S_{Y}^{n}$.}

The interval $I=[-1,1]$ with the antipodal-map is a standard space for the study of involutions and gives rise to the study of the $n$-cube $I^{n}$ and the $n$-sphere $S^{n}$. We introduce a similar space for the study of maps of order 3 . A natural candidate is the tripod, a $Y$-shaped space.

Consider in the complex plane the rotation $\gamma$ of 120 degrees around 0 which is induced by the multiplication with $\zeta=\exp \left(\frac{2 \pi i}{3}\right)$. Let $I$ denote the closed segment between 0 and 1. The subspace $Y=I \cup \zeta(I) \cup \zeta^{2}(I)$ is called the closed $Y$-interval. The points $1, \zeta$ and $\zeta^{2}$ are called the end points of $Y$ and $Y^{\circ}=Y \backslash\left\{1, \zeta, \zeta^{2}\right\}$ is referred to as the open $Y$-interval. Both $Y$ and $Y^{\circ}$ are invariant under $\gamma$. The space $Y^{n}$ is called the $n$-dimensional $Y$-cube. The product map $\gamma^{n}: Y^{n} \rightarrow Y^{n}$ is a map of order 3 with a unique fixed point. The subspace $S_{Y}^{n-1}$ of $Y^{n}$ is defined by

$$
S_{Y}^{n-1}=\left\{\left(x_{1}, \ldots, x_{n}\right) \in Y^{n}: x_{i} \in\left\{1, \zeta, \zeta^{2}\right\} \text { for some } i\right\} .
$$


The space $S_{Y}^{n-1}$ is called the $(n-1)$-dimensional $Y$-sphere. The 1-dimensional $Y$-sphere is the familiar bipartite cubic graph on six nodes $K(3,3)$, which is a standard example of a non-planar graph.

Note that $S_{Y}^{n-1}$ is invariant under $\gamma^{n}$ and that $\gamma^{n}$ has no fixed points in $S_{Y}^{n-1} \cdot \operatorname{As} \operatorname{dim}\left(S_{Y}^{n}\right)=n$ we have

$$
\operatorname{col}\left(S_{Y}^{n}, \gamma^{n+1}\right) \leq n+3 .
$$

We will show later on that $\operatorname{col}\left(S_{Y}^{n}, \gamma\right)=n+3$. So $\left(S_{Y}^{n}, \gamma^{n+1}\right)$ is a pair of an $n$-dimensional space and a mapping for which the color number is maximal. The space $S_{Y}^{n}$ can be obtained from $S_{Y}^{n-1}$ as in the following way

$$
S_{Y}^{n}=\left[S_{Y}^{n-1} \times Y\right] \cup \bigcup_{i=0}^{2}\left[Y^{n} \times\left\{\zeta^{i}\right\}\right] \subset Y^{n+1}
$$

and

$$
S_{Y}^{n}=\left[S_{Y}^{n-1} \times Y^{\circ}\right] \cup \bigcup_{i=0}^{2}\left[Y^{n} \times\left\{\zeta^{i}\right\}\right] \subset Y^{n+1} .
$$

Lemma 1. Let $X$ be a space and $\sigma: X \rightarrow X$ a map of order 3 without fixed-points. Suppose $D$ is a closed subset such that $D \cap \sigma(D)=\emptyset$ and $E=D \cup \sigma(D) \cup \sigma^{2}(D)$, then there exist an equivariant map $f: X \rightarrow Y$ with $f^{-1}(1)=D$.

In the lemma, one may think of $D$ and $E$ as a closed color and closed set of the first kind.

Proof. By enlarging the $\sigma^{i}(D)$ for $i=0,1,2$ we obtain open colors $U_{i}$. For $i=0,1,2$ define real-valued Urysohn functions $f_{i}$ on $X$ such that $0 \leq f_{i}(x) \leq$ 1 for all $x, f_{i}^{-1}(1)=\sigma^{i}(D)$ and $f_{i}^{-1}(0)=X \backslash U_{i}$. Let $f=f_{0}+\zeta f_{1}+\zeta^{2} f_{2}$.

Theorem 3. Let $X$ be a space and $\sigma: X \rightarrow X$ a map of order 3 without fixed-points. The following statements are equivalent:

(1) $\operatorname{col}(X, \sigma) \leq n+3$,

(2) $\operatorname{gen}(X, \sigma) \leq n+1$,

(3) there exist an equivariant map $f:(X, \sigma) \rightarrow\left(S_{Y}^{n}, \gamma^{n+1}\right)$,

(4) $X=\bigcup_{i=1}^{n+1} B_{i}$, where $B_{i}$ is a $\sigma$-invariant subspace with $\operatorname{col}\left(B_{i}, \sigma\right)=3$ for all $i$.

Proof. Suppose (1) holds. Let $\mathcal{A}=\left\{A_{1}, \ldots, A_{n+1}, A_{n+2}, A_{n+3}\right\}$ be an open coloring of $X$. If $x \in A_{n+2} \cup A_{n+3}$ then at least one of the points $\sigma(x)$ and $\sigma^{2}(x)$ does not belong to $A_{n+2} \cup A_{n+3}$, as $\mathcal{A}$ is a coloring of $X$. It follows that

$$
X=\bigcup_{i=1}^{n+1}\left[A_{i} \cup \sigma\left(A_{i}\right) \cup \sigma^{2}\left(A_{i}\right)\right],
$$


whence (2) holds. If $(X, \sigma)$ satisfies (2), then $X=\bigcup_{i=1}^{n+1} B_{i}$ with $B_{i}=$ $C_{i} \cup \sigma\left(C_{i}\right) \cup \sigma^{2}\left(C_{i}\right)$ a set of first type. By shrinking the cover $\left\{\sigma^{j}\left(C_{i}\right)\right.$ : $i=1, \ldots, n ; j=0,1,2\}$ one can find closed subsets $D_{i} \subset C_{i}$ such that $\left\{\sigma^{j}\left(D_{i}\right): i=1, \ldots, n ; j=0,1,2\right\}$ is a cover of $X$. By Lemma 1 there is for each $i$ an equivariant map $f_{i}: X \rightarrow Y$ with the property $f_{i}^{-1}(1)=D_{i}$. The evaluation map $f=\left(f_{1}, \ldots, f_{n+1}\right)$ of $X$ to $Y^{n+1}$ is equivariant and sends $X$ to $S_{Y}^{n}$. So (3) holds. We have already observed that $\operatorname{col}\left(S_{Y}^{n}, \gamma^{n+1}\right) \leq n+3$. Thus (1) follows from (3).

To complete the proof we show that (2) and (4) are equivalent. If $(X, \sigma)$ satisfies (2), then $X=\bigcup_{i=1}^{n+1} B_{i}$ where each $B_{i}=C_{i} \cup \sigma\left(C_{i}\right) \cup \sigma^{2}\left(C_{i}\right)$ is a set of first type. The subspaces $B_{i}$ are invariant and have color number 3 . Now, suppose that (4) holds. For each $i$ let $C_{i}$ be a color of the subspace $B_{i}$ witnessing the fact that $\operatorname{col}\left(B_{i}, \sigma\right)=3$. For each $i$ the set $C_{i}$ is open in the subspace $B_{i}$ and the sets $C_{i}, \sigma\left(C_{i}\right)$ and $\sigma^{2}\left(C_{i}\right)$ are mutually disjoint subsets of $B_{i}$. As the sets $C_{i}, \sigma\left(C_{i}\right)$ and $\sigma^{2}\left(C_{i}\right)$ are mutually separated in $X$ there is an color $U_{i}$ of $(X, \sigma)$ such that $B_{i} \cap U_{i}=C_{i}$. The set $V_{i}=U_{i} \cup \sigma\left(U_{i}\right) \cup \sigma^{2}\left(U_{i}\right)$ is of the first type and $X=\bigcup_{i=1}^{n+1} V_{i}$, whence $\operatorname{gen}(X, \sigma) \leq n+1$.

From the equivalence of (2) and (4) in the previous theorem and the construction of $S_{Y}^{n}$ out of $S_{Y}^{n-1}$ one can obtain

$$
\operatorname{gen}\left(S_{Y}^{n}, \gamma^{n+1}\right) \leq 1+\operatorname{gen}\left(S_{Y}^{n-1}, \gamma^{n}\right) .
$$

To prove this formula let $S_{Y}^{n-1}=\bigcup_{i=1}^{k} B_{i}$, where $B_{i}$ is a $\gamma^{n}$-invariant subspace with $\operatorname{col}\left(B_{i}, \sigma\right)=3$ for all $i$ and $k=\operatorname{gen}\left(S_{Y}^{n-1}, \gamma^{n}\right)$. Then $S_{Y}^{n}=$ $\left[\bigcup_{i=1}^{n} B_{i} \times Y^{\circ}\right] \cup L^{n}$ and $\operatorname{gen}\left(S_{Y}^{n}, \gamma^{n+1}\right) \leq k+1$, where $L^{n}=Y^{n} \times\{1,2,3\}$.

Theorem 4. $\operatorname{col}\left(S_{Y}^{n}, \gamma^{n+1}\right)=n+3$ and $\operatorname{gen}\left(S_{Y}^{n}, \gamma^{n+1}\right)=n+1$.

Proof. It has already been observed that $\operatorname{col}\left(S_{Y}^{n}, \gamma^{n+1}\right) \leq n+3$. It is known that for all odd $n$ the standard sphere $S^{n}$ with the standard map $\sigma$ of period three has color number $n+3$. So there exists an equivariant map $f:\left(S^{n}, \sigma\right) \rightarrow\left(S_{Y}^{n}, \gamma^{n+1}\right)$. It follows that $\operatorname{col}\left(S_{Y}^{n}, \gamma^{n+1}\right) \geq n+3$ and by Theorem 3 one obtains gen $\left(S_{Y}^{n}, \gamma^{n+1}\right)=n+1$. Now suppose that $n$ is even and $\operatorname{col}\left(S_{Y}^{n}, \gamma^{n+1}\right)<n+3$. By Theorem 3 it follows that $\operatorname{gen}\left(S_{Y}^{n}, \gamma^{n+1}\right)<$ $n+1$. Then by the formula (1) we get $\operatorname{gen}\left(S_{Y}^{n+1}, \gamma^{n+2}\right)<n+2$, which cannot be true, as $n+1$ is odd.

\section{Ljusternik-Schnirelmann for maps of order 3 .}

Theorem 2 is similar to the Ljusternik-Schnirelmann theorem which reads as follows. Suppose that $\sigma$ is an involution on a space $X$ and $\operatorname{col}(X, \sigma)=n+2$. Suppose $\left\{A_{1}, \ldots, A_{n+1}, A_{n+2}\right\}$ is a coloring of $X$. Then $\bigcap_{i=1}^{n+1} A_{i} \neq \emptyset$. The reason why this is true follows. If the two points $\mathbf{x}_{1}=(1, \ldots, 1)$ and $\mathbf{x}_{\mathbf{2}}=(-1, \ldots,-1)$ are deleted from the standard sphere $S^{n} \subset[-1,1]^{n+1}$ one 
obtains a space with a strictly smaller coloring number (for $n \geq 1$ ). For maps of order 3 one can not make a similar claim, $\bigcap_{i=1}^{n+1} A_{i} \neq \emptyset$, as easy examples on $S^{1}$ already show. If in the space $S_{Y}^{n}$ the three points $\mathbf{x}_{\mathbf{i}}=\left(\zeta^{i}, \ldots, \zeta^{i}\right)$, $i=0,1,2$, are deleted one obtains a space with the same coloring number. The color number decreases only if more points are deleted.

Consider the $n$-dimensional $Y$-sphere $S_{Y}^{n}$ and define the subset $\Lambda_{Y}^{n}$ by

$$
\mathbf{x}=\left(x_{i}\right)_{i} \in \Lambda_{Y}^{n} \text { if and only if } x_{i} \in\left\{1, \zeta, \zeta^{2}\right\} \text { for all } i .
$$

Note that the cardinality of $\Lambda_{Y}^{n}$ is $3^{n+1}$ and that $\Lambda_{Y}^{n}$ is invariant under $\gamma^{n+1}$. From Theorem 5 it follows that the color number can be reduced if we delete the subset $\Lambda_{Y}^{n}$ from $S_{Y}^{n}$.

We define a subspace $\Sigma^{n} \subset \Lambda_{Y}^{n}$ in the folowing way. For $i=0,1,2$, the subset $\Sigma_{i}^{n}$ of $\Lambda^{n}$ is defined by

$$
\Sigma_{i}^{n}=\left\{\mathbf{x}=\left(x_{j}\right)_{j}: x_{j}=\zeta^{i-1} \text { or } x_{j}=\zeta^{i+1}\right\} \backslash \Delta,
$$

where $\Delta$ is the diagonal of $Y^{n+1}$, i.e., $\Delta$ is the set of points all whose coordinates are equal. For example, $\mathbf{x}=\left(\zeta, \zeta^{2}, \zeta\right) \in \Sigma_{0}^{2}$, but $\mathbf{y}=(\zeta, \zeta, \zeta) \notin \Sigma_{0}^{2}$. Note that the three sets $\Sigma_{i}^{n}, i=0,1,2$, are pairwise disjoint and $\gamma^{n+1}\left(\Sigma_{i}^{n}\right)=$ $\Sigma_{(i+1) \bmod 3}^{n}$. It follows that the set $\Sigma^{n}=\Sigma_{0}^{n} \cup \Sigma_{1}^{n} \cup \Sigma_{2}^{n}$ is $\gamma^{n+1}$-invariant.

Theorem 5. For $n \geq 1$,

$$
\operatorname{col}\left(S_{Y}^{n} \backslash \Sigma^{n}, \gamma^{n+1}\right) \leq n+2 \quad \text { and } \quad \operatorname{gen}\left(S_{Y}^{n} \backslash \Sigma^{n}, \gamma^{n+1}\right) \leq n .
$$

Proof. As the statements of the theorem are equivalent by Theorem 3, we prove the second. For $n=1$ it is best to verify the statement by drawing a picture. There are six points in $\Sigma^{1}$. We mentioned already that $S_{Y}^{1}$ is the graph $K(3,3)$, which is cut by $\Sigma^{1}$ in six of its edges. The three edges that remain intact, connect vertices of two opposite parts of the graph. So $S_{Y}^{1} \backslash \Sigma^{1}$ consists of three components, that are permuted by $\gamma^{2}$. Thus $S_{Y}^{1} \backslash \Sigma^{1}$ is a set of the first type.

Now assume that the result holds for $\left(S_{Y}^{n-1} \backslash \Sigma^{n-1}, \gamma^{n}\right)$. As $S_{Y}^{n}=\left[S_{Y}^{n-1} \times\right.$ $\left.Y^{\circ}\right] \cup \bigcup_{i=0}^{2}\left[Y^{n} \times\left\{\zeta^{i}\right\}\right]$ and $\left(S_{Y}^{n-1} \times Y^{\circ}\right) \cap \Sigma^{n}=\emptyset$, we have

$$
\begin{aligned}
S_{Y}^{n} \backslash \Sigma^{n}=\left[\left(S_{Y}^{n-1} \backslash \Sigma^{n-1}\right) \times Y^{\circ}\right] & \cup \\
& {\left[\left(\Sigma^{n-1} \times Y^{\circ}\right) \cup \bigcup_{i=0}^{2}\left\{\left(Y^{n} \times\left\{\zeta^{i}\right\}\right) \backslash \Sigma^{n}\right\}\right] . }
\end{aligned}
$$

The induction hypothesis implies that gen $\left(\left(S_{Y}^{n-1} \backslash \Sigma^{n-1}\right) \times Y^{\circ}, \gamma^{n+1}\right) \leq n-1$. So it suffices to show that the remaining two sets $\left(\Sigma^{n-1} \times Y^{\circ}\right) \cup \bigcup_{i=0}^{2}\left(Y^{n} \times\right.$ $\left.\left\{\zeta^{i}\right\}\right) \backslash \Sigma^{n}$ form a set of genus 1 . Define the sets $A_{i}$ for $i=0,1,2$ by

$$
A_{i}=\left(\Sigma_{i}^{n-1} \times Y^{\circ}\right) \cup\left(Y^{n} \times\left\{\zeta^{i}\right\}\right) \backslash \Sigma^{n} .
$$


One verifies that this set has the property $\gamma^{n+1}\left(A_{i}\right) \cap A_{i}=\emptyset$ and that $\left(\Sigma^{n-1} \times Y^{\circ}\right) \cup \bigcup_{i=0}^{2}\left(Y^{n} \times\left\{\zeta^{i}\right\}\right) \backslash \Sigma^{n}=A_{0} \cup A_{1} \cup A_{2}$. So the proof is finished once we verify that $A_{0}$ is a closed subset of $S_{Y}^{n} \backslash \Sigma^{n}$. It is obvious that $Y^{n} \times\{1\}$ is closed, so we have to verify that the closure of $\Sigma^{n-1} \times Y^{\circ}$ is contained in $A_{0}$. Observe that the set of density points of $\Sigma_{0}^{n-1} \times Y^{\circ}$ in the $Y$-sphere is $\Sigma_{0}^{n-1} \times\left\{1, \zeta, \zeta^{2}\right\}$. So in $S_{Y}^{n} \backslash \Sigma^{n}$ its set of density points is $\Sigma_{0}^{n-1} \times\{1\}$, which is a subset of $Y^{n} \times\{1\}$. We conclude that $A_{0}$ is closed in $S_{Y}^{n} \backslash \Sigma^{n}$.

The previous theorem has as a corollary Theorem 2 .

Proof. Note first that the three statements

(1) $\bigcap_{i=1}^{n+1}\left[A_{i} \cup \sigma\left(A_{i}\right)\right] \neq \emptyset$,

(2) $\bigcap_{i=1}^{n+1}\left[\sigma\left(A_{i}\right) \cup \sigma^{2}\left(A_{i}\right)\right] \neq \emptyset$,

(3) $\bigcap_{i=1}^{n+1}\left[\sigma^{2}\left(A_{i}\right) \cup A_{i}\right] \neq \emptyset$,

are equivalent, as these sets are mapped onto each other by the map $\sigma$. Using the closed coloring $\left\{A_{1}, \ldots, A_{n+1}, A_{n+2}, A_{n+3}\right\}$, for $i=1, \ldots, n+1$, we can define by Lemma 1 equivariant maps $f_{i}: X \rightarrow Y$ with $f_{i}^{-1}(1)=A_{i}$. The evaluation map $F=\left(f_{1}, \ldots, f_{n+1}\right): X \rightarrow Y^{n+1}$ is equivariant and $F(X) \subset S_{Y}^{n}$. Since $\operatorname{col}(X, \sigma)=n+3$ and $\operatorname{col}\left(S_{Y}^{n} \backslash \Sigma_{n}, \gamma^{n+1}\right) \leq n+2$, it can not occur that $F(X) \subset S_{Y}^{n} \backslash \Sigma^{n}$. Choose $x \in X$ with $F(x)=\left(f_{i}(x)\right)_{i} \in \Sigma^{n}$, say $F(x) \in \Sigma_{1}^{n}$.

It follows that $x \in \bigcap_{i=1}^{n+1}\left[A_{i} \cup \sigma^{2}\left(A_{i}\right)\right] \neq \emptyset$ and so $\sigma(x) \in \bigcap_{i=1}^{n+1}\left[A_{i} \cup\right.$ $\left.\sigma\left(A_{i}\right)\right] \neq \emptyset$. Similar arguments can be used for the cases $F(x) \in \Sigma_{2}^{n}$ and $F(x) \in \Sigma_{0}^{n}$.

\section{Colorings of non-invariant subspaces.}

We have defined colors as special open subsets. It was already observed that in defining colors we could have used closed sets as well. However, when studying non-invariant subspaces we must stick to open colors.

Definition 3. Suppose that $f: X \rightarrow X$ is a map from $X$ to itself. Let $A$ be a subset of $X$. A coloring of the subset $A$ is a finite collection $\mathcal{B}$ consisting of colors of $(X, f)$ such that $A \subset \cup \mathcal{B}$. We denote the minimal cardinality of such a collection by $\operatorname{col}(A, X, f)$.

With the technique of the proof of Theorem 3, the equivalence of (2) and (4) one can prove the following lemma. We use the notation $\sigma_{B}$ to denote the restriction of the map $\sigma$ to the subspace $B$.

Lemma $2([8])$. Let $X$ be a space and $\sigma: X \rightarrow X$ a map of period 3 without fixed-points. If $A \subset B \subset X$ and $B$ is $\sigma$-invariant, then

$$
\operatorname{col}(A, X, \sigma)=\operatorname{col}\left(A, B, \sigma_{B}\right) .
$$


It is a consequence of the lemma that to compute the color number of a subset $A$ of $X$, we may assume that $X=A \cup \sigma(A) \cup \sigma^{2}(A)$. The following theorem provides an upper bound for the color number of a subset related to its dimension.

Theorem 6. Suppose that $\sigma: X \rightarrow X$ is a map of period 3 without fixedpoints. If $A$ is a subset of $X$ and $\operatorname{dim}(A) \leq n$ then $\operatorname{col}(A, X, \sigma) \leq 3 n+5$.

Proof. The $\sigma$-invariant subspace $T=A \cup \sigma(A) \cup \sigma^{2}(A)$ has dimension at most $3 n+2$ and contains no fixed points of $\sigma$. So $\operatorname{col}\left(T, \sigma_{T}\right) \leq \operatorname{dim}(T)+3=3 n+5$. The result follows from the previous lemma.

We shall present an example of a map $\sigma$ of period 3 with $\operatorname{col}(A, X, \sigma) \geq$ $3 n+4$. We refer to [8] for a related example of an involution $\iota$ with color number $\operatorname{col}(A, X, \iota)=2 n+3$. For the construction we need the following result, which is a consequence of Theorem 1 .

Theorem 7. Let $\sigma: X \rightarrow X$ be a map of period 3 without fixed-points. Suppose $\operatorname{col}(X, \sigma)=n+3$. If $A$ is a dense subset of $X$ with $A \cup \sigma(A) \cup$ $\sigma^{2}(A)=X$ then $\operatorname{col}(A, \sigma) \geq n+2$.

Proof. We argue by contradiction. Assume that $\left\{U_{1}, \ldots, U_{n+1}\right\}$ is a coloring of $(A, X, \sigma)$. Then $X=\bigcup_{i=1}^{n+1}\left(U_{i} \cup \sigma\left(U_{i}\right) \cup \sigma^{2}\left(U_{i}\right)\right)$. As $\operatorname{col}(X, \sigma)=n+3$, we have $\operatorname{gen}(X, \sigma)=n+1$. From Theorem 1 it follows that $\bigcap_{i=1}^{n+1}\left[\sigma^{-1}\left(U_{i}\right) \cup\right.$ $\left.\sigma\left(U_{i}\right)\right]$ is a nonempty open set, which by the density of $A$ contains an element $a \in A$. For each index $i$ we have either $\sigma(a) \in U_{i}$ or $\sigma^{-1}(a) \in U_{i}$ and therefore $a \notin U_{i}$. This contradicts that the $U_{i}$ cover $A$.

For the construction of our example we need the following lemmas. The first lemma is a special case of a result in [4].

Lemma 3. Let $X$ be a space of dimension 2 with a fixed-point free map $\sigma: X \rightarrow X$ of period 3. Then there exists a subspace $A$ of $X$ with the following properties

(1) $A$ is dense in $X$,

(2) $\operatorname{dim} A=0$,

(3) $A \cup \sigma(A) \cup \sigma^{2}(A)=X$.

The following lemma follows easily from the fact that a subset of $X$ is contained in a $G_{\delta}$-subset of the same dimension.

Lemma 4. Let $X$ be a space with a fixed-point free map $\sigma: X \rightarrow X$ of period 3. If $A$ is a $\sigma$-invariant subspace of $X$ with $\operatorname{dim}(A)=k$ then there exists a $\sigma$-invariant $G_{\delta}$ subset $A^{\prime}$ of $X$ with $A \subset A^{\prime}$ and $\operatorname{dim} A^{\prime}=\operatorname{dim} A$.

Lemma 5. Let $X$ be a space with $\operatorname{dim} X=n$ and with a fixed-point free map $\sigma: X \rightarrow X$ of period 3. Then $X=X_{0} \cup \ldots \cup X_{n}$, where $\operatorname{dim} X_{i}=0$ and each $X_{i}$ is $\sigma$-invariant. 
Proof. This is a version of the Decomposition Theorem [11]. We use induction on the dimension. If $\operatorname{dim} X=0$ the result is trivial. Assume the result holds for all spaces of dimension $\leq k-1$ and all maps of order 3 without fixed-point. Let $X$ be a space with $\operatorname{dim} X=k$ and let $\sigma: X \rightarrow X$ be a map of period 3 without fixed-points. Let $\left\{U_{n} ; n \in \omega_{0}\right\}$ be a countable base with $\operatorname{dim}\left(\operatorname{cl} U_{n} \backslash U_{n}\right) \leq k-1$, for all $n$. If we define

$$
X_{k}=X \backslash\left[\bigcup_{n}\left(\operatorname{cl} U_{n} \backslash U_{n}\right) \cup \bigcup_{n} \sigma\left(\operatorname{cl} U_{n} \backslash U_{n}\right) \cup \bigcup_{n} \sigma^{2}\left(\operatorname{cl} U_{n} \backslash U_{n}\right)\right]
$$

then $X_{k}$ is $\sigma$-invariant, $\operatorname{dim} X_{k}=0$ and $\operatorname{dim}\left(X \backslash X_{k}\right) \leq k-1$. So the induction argument applies.

Finally we construct the example of a complete $n$-dimensional subspace that cannot be colored with less than $3 n+4$ colors.

Example 1. Consider the space $S_{Y}^{3 n+2}$ with the standard map $\gamma: S_{Y}^{3 n+2} \rightarrow$ $S_{Y}^{3 n+2}$ of order 3 . We know that $\operatorname{col}\left(S_{Y}^{3 n+2}, \gamma\right)=3 n+5$. Since the $S_{Y}^{3 n+2}$ is $(3 n+2)$-dimensional it can be written as the union of $3 n+3$ zero-dimensional subspaces, say $S_{Y}^{3 n+2}=B_{1} \cup \ldots \cup B_{3 n+3}$ such that each $B_{i}$ is zero-dimensional and $\gamma$-invariant. Let $X_{i}=B_{3 i-2} \cup B_{3 i-1} \cup B_{3 i}$ for $i=1, \ldots, n+1$. Then each $X_{i}$ is $\leq 2$-dimensional, invariant under $\gamma$. Lemma 3 implies that each $X_{i}$ has a dense zero-dimensional subspace $A_{i}$ such that $A_{i} \cup \gamma\left(A_{i}\right) \cup \gamma^{2}\left(A_{i}\right)=X_{i}$. Write $A^{\prime}=A_{1} \cup \ldots \cup A_{n+1}$. Then, $A^{\prime}$ is dense, $A^{\prime}$ has dimension $\leq n$ and $A^{\prime}$ also has the property

$$
A^{\prime} \cup \gamma\left(A^{\prime}\right) \cup \gamma 2\left(A^{\prime}\right)=S_{Y}^{3 n+2} .
$$

Note that Property (2) implies that $\operatorname{dim} A^{\prime}=n$. We enlarge $A^{\prime}$ to a dense $G_{\delta}$ subset $A$ of dimension $n$. Then $A$ satisfies Property (2). Finally, by Theorem 7 we have $\operatorname{col}(A, X, \gamma) \geq 3 n+4$.

\section{Some remarks on periodic maps.}

For a general fixed-point free homeomorphism $f: X \rightarrow X$ we were unable to obtain bounds on $\operatorname{col}(A, X, f)$ in terms of $\operatorname{dim} A$. Indeed, we do not even know whether such a bound exists. However, we can find such a bound in the special case that the map is periodic.

We need the following result of Steinlein.

Theorem 8 ([15]). If $f: S^{(m-1)(p-1)-1} \rightarrow S^{(m-1)(p-1)-1}$ is a fixed-point free map of prime-period $p$, then

$$
\operatorname{col}\left(S^{(m-1)(p-1)-1}, f\right) \leq 4 m .
$$

To obtain our final result, one should know that any free periodic homeomorphism $f: X \rightarrow X$ on a space of $\operatorname{dim} X=n$ can be conjugated to 
a free periodic homeomorphism on $S^{n}$ [15], so that it suffices to consider homeomorphisms on $S^{n}$.

Theorem 9. Let $\sigma: X \rightarrow X$ be a map of prime-period $p$, without fixedpoints and suppose that $p-1$ divides $n+1$. If $A$ is a $n$-dimensional subset of $X$ then $\operatorname{col}(A, X, \sigma) \leq 5 n+12$.

Proof. Suppose that $f: X \rightarrow X$ is a map of order $p$ and let $A$ be subspace of $X$ of dimension $n$. We can assume that $p \geq 5$, since the case $p=2$ is done in [8] and $p=3$ is done above. As we can assume that $X=$ $A \cup f(A) \cup \cdots \cup f^{p-1}(A)$, we see that the maximal dimension of $X$ is $p n+p-1$. Choose a minimal $m$ such that $\operatorname{dim}(X) \leq(m-1)(p-1)-1$ and so

$$
p n+p-1 \leq(m-1)(p-1)-1 .
$$

This implies that we can take

$$
m=\left\lceil\frac{p n+p}{p-1}+1\right\rceil .
$$

We conclude that we can color $X$, hence $A$, with $4 m=4\left\lceil\frac{p n+p}{p-1}+1\right\rceil \leq 5 n+12$ colors.

\section{References}

[1] J.M. Aarts, G.A. Brouwer, R.J. Fokkink and J. Vermeer, Intersection properties of coverings of $G$-spaces, to appear in Topology Appl.

[2] J.M. Aarts and R.J. Fokkink, An addition theorem for the color number, Proc. Amer. Math. Soc., 129(9) (2001), 2803-2807 (electronic), CMP 1838 806, Zbl 0963.54026.

[3] J.M. Aarts, R.J. Fokkink and J. Vermeer, Variations on a theorem of Lusternik and Schnirelman, Topology, 35 (1996), 1051-1056, MR 98e:55003, Zbl 0918.54011.

[4] _ A dynamic decomposition theorem, to appear in Acta Math. Hungar., 94(3), (2002).

[5] A. Błaszczyk and J. Vermeer, Some old and some new results on combinatorial properties of fixed-point free maps, Ann. New York Acad. Sci., 767 (1995), 1-16, MR 98g:54100, Zbl 0919.54034.

[6] A. Błaszczyk and Kim Dok Yong, A topological version of a combinatorial theorem of Katětov, Com. Math. Univ. Car., 29 (1988), 657-663.

[7] J. Dugundji and A. Granas, Fixed Point Theory, Polish Scientific Publishers, Warszawa, 1988, MR 83j:54038, Zbl 0483.47038.

[8] J.A.M. de Groot and J. Vermeer, Liusternik, Schnirelmann for subspaces, Topology Appl., 115(3) (2001), 343-354, CMP 1848134.

[9] M. van Hartskamp, Colorings of fixed-point free maps, Thesis, Free University, Amsterdam, 1999.

[10] M. van Hartskamp and J.Vermeer, On colorings of maps, Topology Appl., 73 (1996), 181-190, MR 97i:55006, Zbl 0867.55003. 
[11] W. Hurewicz and H. Wallman, Dimension Theory, Princeton University Press, Princeton NJ, 1941, MR 3,312b, Zbl 0060.39808.

[12] M.A. Krasnoselski, On special coverings of a finite dimensional sphere, Dokl. Akad. Nauk SSSR, 103 (1955), 961-964 (Russian), MR 18,406a.

[13] K. Kuratowski, Topology I, Academic Press, 1966, MR 36 \#840, Zbl 0158.40802.

[14] J. van Mill, Easier proofs of coloring theorems, Topology Appl., 97 (1999), 155-163, MR 2000i:54066, Zbl 0947.54019.

[15] H. Steinlein, On the theorems of Borsuk-Ulam and Ljusternik-Schnirelmann-Borsuk, Canad. Math. Bull., 27 (1984), 192-204, Zbl 0531.55002.

[16] H. Steinlein, Borsuk's antipodal theorem and its generalizations and applications: A survey, in 'Methodes topologiques en Analyse non lineaire', les presses de l'universite de Montreal, A.Granas, 1985, MR 86k:55002, Zbl 0573.55003.

Received May 16, 2000 and revised August 29, 2000.

\author{
Technical University Delft \\ FACULTY I.T.S. \\ P.O. Box 5031 \\ 2600 GA DELFT \\ The Netherlands \\ E-mail address: Aarts@its.tudelft.nl \\ Technical University Delft \\ FACULTY I.T.S. \\ P.O. Box 5031 \\ 2600 GA DelfT \\ The Netherlands \\ E-mail address: fokkink@its.tudelft.nl \\ Technical University Delft \\ FACUlty I.T.S. \\ P.O. Box 5031 \\ 2600 GA DELFT \\ The NeTHERlands \\ E-mail address: J.Vermeer@its.tudelft.nl
}

